\title{
Estabilidad de la posición de una facción e influencia
}

\section{Charlan Nemeth}

FuENTE: Brandstatter, H. et al. (eds.) Group Decision Making, Londres: Academic, 1982.

En los clásicos estudios de Festinger sobre las presiones a la uniformidad en el grupo, se hizo patente que la gente supone que la verdad descansa en el consenso y que la disidencia es una situación desagradable que provoca presiones en favor del acuerdo. A partir de la década de 1950 y el clásico trabajo de Asch, un importante monto de la investigación se ha concentrado en el modo en que puede lograrse tal consenso. Es decir, en el proceso de la conformidad. En esta situación, el desviado o la minoría modifican su posición en la dirección de la posición de la mayoría, evidenciando así un proceso de control social.

Los estudios que prosiguieton en las décadas posteriores acumularon una cantidad considerable de datos, demostrando repetidas veces que la causa de la incomodidad de la situación era el desacuerdo, y que la gente «se esfuerza por conseguir uniformidad».

Deutsch y Gerard (1955), por ejemplo, aclararon estas nociones básicas con su distinción entre «influencia normativa» $\mathrm{e}$ «informativa». La influencia normativa, según Deutsch y Gerard, es «una influencia para conformarse respecto a las expectativas positivas de otro». Dicho de otro modo, algunos su- jetos pueden conformarse porque anticipan las recompensas y ausencia de castigos provenientes de los demás. La influencia informativa es, por su parte, definida como «una influencia que lleva a aceptar la información proveniente de otro como una evidencia sobre la realidad».

El malestar que produce la disidencia queda demostrado por los informes de los sujetos de Asch que no deseaban «llamar la atención como un burro en una cacharrería», y nuestra propia investigación (Nemeth, 1976; Wachtler y Nemeth, 1978) en la que. las minorías disidentes respecto a una mayoría acusan un considerable stress.

La premisa fundamental de la influencia informativa es el supuesto de que la verdad descansa en el consenso. Cuando no es posible determinar físicamente la verdad o falsedad de una posición, la gente confía en los demás para decidir si sus percepciones $u$ opiniones son correctas. Así, algunos de los sujetos de Asch indicaron preocupación por sus propias opiniones y juicios, ya que los demás disentían: «no pueden estar todos equivocados y yo en lo cierto». La duda acerca de los propios juicios se debió presumiblemente a la creencia de que la 
verdad se basa en el acuerdo; cuando hay desacuerdo hay presiones en pro del consenso para validar las opiniones propias.

Durante la década actual ha despertado mucho interés otra de las formas en que puede lograrse el consenso, esto es, la influencia de la minoría. En este caso, el proceso consiste en que la mayoría cambia su posición en la dirección de aquella a la que se adhiere la minoría. Una vez más encontramos pruebas del malestar que engendra el desacuerdo y las presiones uniformadoras. Pero ahora el proceso implicado y las formas concretas de malestar y presión son diferentes. En realidad la minoría parece lograr la aceptación de su pusición fomcritando el malestar que implica el desacuerdo y contando con las presiones concomitantes en favor de la uniformización y el consenso. En muchos estudios (Moscovici et al, 1969; Nemeth y Wachtler, 1974), lo que es necesario para la influencia minoritaria es precisamente que la minoría mantenga su posición; el mantenimiento del desacuerdo, de la viabilidad de una postura alternativa, es lo que crea por parte de la mayoría las presiones consensualizadoras y el malestar necesarios que sirven para hacer influyente la postura minoritaria.

En trabajos anteriores hemos comentado algunas de las principales diferencias entre el proceso de conformidad y la influencia minoritaria (Nemeth, 1976, 1978; Wachtler y Nemeth, 1978). Una de las diferencias es que la conformidad a menudo se observa en la primera prueba experimental o durante los primeros momentos de una discusión. Por el contrario, es preciso más tiempo para que se manifieste una influencia minoritaria, rara vez se observa en las primeras pruebas experimentales o durante los primeros minutos de una discusión. En segundo lugar, la oposición en las situaciones de conformidad, esto es, la mayoría, resulta mucho más simpática que la oposición en la situación de influencia minotitaria. Rara vez se tiene simpatía al desviado o a la minoría insistente; en realidad, se les tiene una gran aversión.
En tercer lugar, el proceso de conformidad parece implicar más stress por parte del sujeto ingenuo (es decir, la minoría) que el que supone el proceso de influencia minoritaria (donde los sujetos ingenuos son una mayoría). Tales altos niveles de stress en el proceso de conformidad parecen motivar a los sujetos ingenuos para que se concentren en la averiguación de quien está en lo cierto: ellos o la mayoría disidente. Por el contrario, el proceso de influencia minoritaria parece caracterizarse por la existencia de un conflicto suficiente como para que la mayoría «reconsidere» su posición, pero no lleva a que sus miembros reduzcan su universo de alternativas a dos: la suya c la de la minoría. Hay más bien evidencias (Nemeth, Wachtler, 1981) de que la persistencia de la posición minoritaria motiva a la mayoría para hallar nuevas alternativas no sugeridas por la minoría ni consideradas previamente por la mayoría. Parece haber, pues, una contribución creativa del conflicto engendrado por el mantenimiento de una posición minoritaria.

En este escrito nos centraremos, en cambio, en las similitudes entre los fenómenos de conformidad e influencia minoritaria y, claro está, entre los procesos de influencia en general. En concreto, sostenemos que gran parte de la literatura acerca de cada uno de estos procesos indica que lo que dictará si el proceso es de conformidad, influencia minoritaria o compromiso, es la estabilidad del «anclaje» o posición propuesta por la mayoría o la minoría. Por último, esbozaremos un estudio recientemente concluido que refuerza la estabilidad del «anclaje» por medio de otro fenómeno psicosocial, i. e., el proceso de polarización. Predecimos distintos resultados de la interacción mayoría-minoría según el bando fortalecido por dicho proceso.

En primer lugar, consideremos la literatura existente acerca de la estabilidad de la conducta como factor de influencia. Los estudios sobre conformidad han centrado su atención, por lo general, en la estabilidad entre personas, esto es, en la consistencia ínter-individual. La mayo- 
ría comienza con varios sujetos en su bando y su grado de unanimidad parece dictar la importancia de su influencia. La mayor parte de los escritos disponibles (Asch, 1956; Rosenberg, 1961; Goldberg, 1954) indican que la conformidad puede aumentar a medida que aumenta el número de sujetos de la mayoría de 1 a 2 y de 2 a 3, pero, a partir de 3, los aumentos numéricos no parecen tener consecuencias importantes. Así pues, el tamaño no parece ser un factor determinante del potencial de conformidad. Por otra parte, la unanimidad parece ser un factor muy importante.

En los estudios iniciales de Asch (1955) se halló que el poder de la mayoría se quebraba con la presencia de un disidente además del sujeto ingenuo. En una de las variaciones de su experimento, este aliado era otro sujeto ingenuo; en una segunda variación se trataba de un ayudante del experimentador al que se le instruyó para dar las respuestas correctas a lo largo de las pruebas. En ambas condiciones, la cantidad de conformidad manifestada por el sujeto ingenuo disminuyó en una proporción de 4 a 1 . Sin embargo, otras condiciones experimentales señalaban el hecho de que no era el apoyo social per se lo que disminuía el impacto de la mayoría, sino más bien el hecho de que la posición mayoritaria había sido quebrantada, es decir, hubo una ruptura de la unanimidad. En una condición experimental esta ruptura la provocó un ayudante del experimentador que respondió de forma incorrecta, pero a mitad de camino entre la posición del sujeto ingenuo y las respuestas incorrectas de la mayoría; en otra condición el ayudante respondió de forma aún más incorrecta que la mayoría. Ambas condiciones provocaron la disminución de la conformidad; en realidad, el disidente extremo provocó una reducción aún mayor de la conformidad, disminuyendo los errores a sólo un 9 por 100. Asch llegó a la conclusión de que «la disidencia per se aumenta la independencia y aminora el número de errores producidos y que la dirección de la disidencia ejerce efectos consistentes» (p. 34).

Suponemos que la estabilidad de la posición propuesta por la mayoría disminuye cuando hay una ruptura en los juicios ofrecidos por los otros. En contraste con una mayoría unánime, la presencia de otro individuo que disiente de la mayoría, esté o no de acuerdo con el sujeto minoritario, disminuye la probabilidad de un consenso estable en torno a la posición mayoritaria. Así, los sujetos pueden tener más probabilidades de mantener su propia opinión.

La prueba más importante en favor de tal argumentación también proviene de otra de las condiciones experimentales diseñadas por Asch. La deserción de un aliado parece aumentar la probabilidad de la conducta conformista. En uno de sus experimentos Asch hacía que un supuesto sujeto experimental (ayudante del experimentador) apoyara las correctas posiciones del sujeto ingenuo a lo largo de seis pruebas; en la séptima prueba el aliado se pasaba a la mayoría. La conformidad aumentaba abruptamente tras la sexta prueba, alcanzando un nivel próximo al de la condición experimental en la que los sujetos se enfrentaban a una mayoría unánime en todas las pruebas.

Sospechamos que la deserción puede haber sido adicionalmente útil para la mayoría, puesto que puede haber dado credibilidad a la posición de la mayoría. A Asch le sorprendió que seis pruebas con apoyo social no «fortalecieran la independencia de los individuos» (p. 34). Parece que sí la fortaleció si tenemos en cuenta el hecho de que cuando el apoyo social simplemente abandonó la habitación (en lugar de pasarse al otro bando), «el efecto del compañero duró más tiempo que su presencia» (p. 34). Así, bien podría ser que la deserción sea más determinante de la conformidad que la mayoría unánime que ya existía anteriormente, puesto que el sujeto debe superar la conformidad respecto a sus propios juicios establecidos durante el período de apoyo social. Nosotros establecemos la hipótesis de que la deserción del aliado 
aumenta la confianza en la posición de la mayoría y, además, muestra bajo una luz poco favorable la posición de la minoría. Además hace aún más evidente que el consenso con mayores probabilidades de ser estable, gire en torno a la posición de la mayoría.

En la influencia minoritaria no se ha hecho tanto énfasis en la consistencia ínter-individual como en la consistencia intra-individual. Los datos existentes muestran que la influencia minoritaria rara vez es evidente durante las primeras pruebas experimentales o en los momentos iniciales de la discusión; en realidad, el desviado o la minoría son, especialmente en estos primeros instantes, objetos habituales de irrisión. Asch (1952) refiere risas de un grupo de dieciséis sujetos ingenuos cuando un individuo manifestó juicios incorrectos discordantes. Schacter (1951) señala que la mayor parte de la comunicación del grupo se dirige hacia el desviado en un intento de cambiar su opinión; cuando esto no se logra, la mayoría reacciona rechazando sociométricamente a la persona desviada. En uno de nuestros estudios (Nemeth y Wachtler, 1974) los sujetos ingenuos ridiculizaron y se rieron de un cómplice del experimentador que sostenía, en un juicio simulado por daños a personas, una posición favorable a una indemnización escasa. Uno de los sujetos ingenuos amenazó al disidente con dañarlo físicamente para demostrarle el «dolor y sufrimiento» implicados en el caso.

Al principio la minoría no es eficaz ni convincente, sino que es justamente su conducta a lo largo del tiempo la orquestación y pautado de señales verbales y no verbales, durante la exposición de su posición, lo que parece hacerla eficaz. $\mathrm{El}$ aspecto más importante de tal patrón y representación de la posición minoritaria parece ser la consistencia, el que se sostenga la posición a lo largo del tiempo.

Esta consistencia intra-individual, la estabilidad de la posición propuesta, es necesaria, aunque no siempre suficiente, para la influencia minoritaria. Moscovici et al (1969) demostraron que una mino- ría de dos sujetos que afirmaron repetidamente que veían "verde» ante diapositivas azules fueron imitados un 8,42 por 100 de las veces. Una minoría que dijo «verde» en $2 / 3$ de las diapositivas y «azul» en $1 / 3$ de las diapositivas fue ineficaz: los sujetos ingenuos juzgaron los estímulos como «azules». Estudios llevados a cabo posteriormente (por ejemplo, Nemeth et al, 1974) demostraron que lo esencial en la influencia minoritaria no era la repetición per se, sino la percepción de que la minoría estaba totalmente convencida de que su posición era la correcta. Las respuestas «azul verdoso» en la mitad de las pruebas experimentales y de «verde» en la otra mitad induce un incremento significativo de la influencia minoritaria cuando las respuestas guardaban relación con una propiedad del estímulo. Cuando no había tal relación (es decir: ambos tipos de respuestas se emitían al azar), la posición de la minoría era percibida como inconsistente y no existió influencia. De hecho, la condición experimental de «correlación» entre las respuestas y alguna propiedad del estímulo era tan eficaz como sostener constantemente la respuesta «azul verdoso», y más eficaz que mantener sin interrupción la respuesta «verde». El aspecto más importante del estilo conductual de la minoría parece radicar en que se les perciba adscritos a una posición consistente, estable y de la que estén convencidos. Conductas más sutiles que la mera repetición, pueden fomentar tal percepción. Sin embargo, una vez más parece que un proceso en el que el consenso gire en torno a la posición minoritaria requiere que dicha posición sea estable, es decir: consistente, a lo largo del tiempo.

Hay otras pruebas en favor de tal planteamiento: si se analiza el proceso de influencia minoritaria a lo largo del tiempo se comprueba que una «ruptura» en la mayoría es una ayuda a la posición defendida por la minoría. Sugerimos que ello induce a percibir que puede ser más estable un consenso en torno a la posición minoritaria que un consenso en 
torno a la posición mayoritaria. Nuestros datos muestran repetidamente un «efecto grupal». Los sujetos ingenuos que constituyen la mayoría se parecen más entre sí de lo que se parecen a los miembros de otras mayorías. Además, hallamos un efecto principal entre las dos mitades para los resultados de la influencia. En términos descriptivos, sería un proceso de ridiculización durante las pruebas iniciales; si la minoría sostiene su posición, algún miembro de la mayoría puede dudar, o lo que es más importante, cambiar su posición en la dirección de la minoría; cuando esto ocurre parece producirse un proceso «en cadena», esto es, los demás miembros de la mayoría comienzan a cambiar su posición en la dirección de la minoría inicial.

Así pues, sugerimos que cada bando debe, en primer lugar, sostener su posición constantemente $\mathrm{y}$, en segundo lugar, mantener la concordancia de sus miembros entre sí. Por otra parte, ello exige resistencia a los intentos de influencia de los demás y el apoyo activo de la posición propia.

$\mathrm{El}$ punto anterior recibe apoyo de un estudio que hemos llevado a cabo utilizando mayorías y minorías naturales (Nemeth, 1977). En dicho estudio realizamos un pretest solicitando a los sujetos que emitieran un veredicto ante un caso de derecho penal que implicaba la acusación de asesinato en primer grado. Solicitamos la participación en un jurado de los individuos que manifestaron claramente un veredicto de culpabilidad o de inocencia, de tal forma que la primera votación se escindiera en una proporción de 4 contra 2. En la mitad de los grupos formados era la minoría la que creía en la inocencia del procesado y en la otra mitad la minoría era partidaria del veredicto de culpabilidad.

La elaboración de este estudio guarda relación con una reciente decisión del Tribunal Supremo de E.E. U.U. con respecto a la tolerancia para con los veredictos no unánimes de un jurado. Para decirlo abiertamente, los estados de Oregón y Louisiana consienten veredictos no unánimes, e individuos convictos por veredictos no unánimes pusieron dos pleitos contra estos estados. La base jurídica fue la presunta violación de los derechos de los acusados según la decimocuarta enmienda de la Constitución de los EE. UU. Los conceptos clave a los que se acogieron fueron «un proceso adecuado» e «igual protección ante la ley».

En la decisión sobre el asunto en litigio, el Tribunal Supremo se dividió en 5 frente a 4. Cinco jueces decidieron que tolerar la no-unanimidad no violó los derechos constitucionales de los procesados, mientras que cuatro jueces disentían de tal resolución. Sin embargo, como el Tribunal Supremo se rige por la decisión de la mayoría, prevaleció la postura de los cinco jueces. Las diferencias de opinión en este caso implican la consideración de diversas teorías sobre la influencia social y las interacciones entre mayorías y minorías en una situación resolutoria. Estas teorías y los resultados empíricos de nuestras investigaciones relacionados con tal decisión pueden hallarse en Nemeth (1977) y Nemeth $e t$ al. (1976).

En resumen, la preocupación de todos los jueces es que hubiera una consideración justa y exhaustiva de todos los puntos de vista, mayoritarios y minoritarios. La opinión del Tribunal Supremo (cinco jueces) fue que la mayoría no impone su voluntad a una minoría cuyos votos no precisa para un veredicto con tal que la minoría haya podido argumentar en favor de su postura. Los cuatro jueces discrepantes temían tal imposición de la voluntad mayoritaria, aduciendo que eran necesarias ciertas garantías. Además, les inquietaba la posible falta de solidez de la deliberación del jurado en caso de que no se exigiese unanimidad.

Nuestro estudio de este caso implicó la modificación de las instrucciones a la mayoría y la minoría y la creación de grupos que tuvieron cuatro opiniones mayoritarias y dos minoritarias. Además, llevamos a cabo un estudio en el que se utilizó un conjunto de juicios simulados en una sala de justicia auténtica con un 
juez presidiendo, comparecencia de testigos, etc. Se consideraron casos de derecho civil y criminal y el «jurado» de doce personas se dividió aleatoriamente en dos grupos de seis personas: en uno de los grupos se exigía un veredicto por unanimidad y en el otro por mayoría.

Sin bien no es relevante tratar aquí los aspectos legales concretos del caso y el problema del requisito de mayoría o unanimidad, sí lo es considerar los procesos de influencia derivados de la relación entre mayoría y minoría. Para que la investigación sobre influencia minoritaria no nos induzca a percibir a las minorías tan eficaces como la mayoría, debemos tener presente que la mayor parte de las decisiones se toman de acuerdo con la dirección de la mayoría inicial. El trabajo clásico de Kalven y Zeisel (1966), en el que se comparan las decisiones de jueces y jurados, señala que más del 90 por 100 de los veredictos definitivos de un jurado se toman según la dirección de la posición mantenida por la mayoría de los miembros del jurado en la primera votación. Nuestro estudio mostró resultados muy similares. En los simulacros del tribunal, todos los grupos, independientemente de que se les solicitara unanimidad o mayoría y de que deliberaran sobre un caso de derecho civil o criminal, dieron un veredicto en la dirección de la posición sostenida por la mayoría de los miembros del jurado en la primera votación.

El único caso en que se hizo sentir influencia minoritaria fue en la situación experimental, concretamente cuando la minoría optó por el veredicto de «inocente» en el caso de asesinato en primer grado. Este experimento implicó 37 grupos que deliberaban sobre el mismo caso $y$, como puede verse en la tabla 1 , a veces prevaleció la opinión minoritaria cuando ésta defendía el veredicto de inocencia.

TABLA 1

\begin{tabular}{lccc}
\hline & $\begin{array}{c}\text { Minoría } \\
\text { «ganadora» }\end{array}$ & $\begin{array}{c}\text { Mayoría } \\
\text { «ganadora» }\end{array}$ & $\begin{array}{c}\text { Pendiente } \\
\text { de veredicto }\end{array}$ \\
\hline $\begin{array}{l}\text { Minoría «inocente» } \\
\text { Mayoría pro «culpable» }\end{array}$ & 7 & 7 & 5 \\
$\begin{array}{l}\text { Minoría «inocente» } \\
\text { Mayoría «culpable» }\end{array}$ & 1 & 16 & 1 \\
\hline
\end{tabular}

Algunos de los datos obtenidos en este estudio son sugerentes aun cuando sólo podemos comparar mayorías y minorías vencedoras cuando la mayoría mantiene el veredicto de culpabilidad y la minoría el de inocencia. Las cintas magnetofónicas de la deliberación se codificaron según quien habló, a quien se dirigió y la naturaleza del comentario de acuerdo con las categorías de Bales.

Comparando los grupos en los que se impuso la minoría con los grupos en que se impuso la mayoría se analizaron los datos observándose qué categorías de conducta eran iniciadas por miembros de la mayoría y cuáles por miembros de la minoría; la dirección de las medidas de tendencia central indica que el «bando ganador» hizo más comentarios pertenecientes a las categorías «se muestra afable» (1), «dramatiza» (2), «está de acuerdo» (3), «hace propuestas» (4), «expresa su opinión» (5), «da información» (6) y «no está de acuerdo» (10). El «bando ganador» expresó menos comentarios de las categorías «pregunta la opinión» (8), «da señales de tensión» (11) y «se muestra hostil» (12). Si bien las diferencias entre medias no son significativas, en parte debido al pequeño tamaño de la muestra y gran dispersión de sus puntuaciones, las pautas 
observadas sugieren que el bando ganador manifiesta una cantidad relativamente superior de comentarios encuadrables en categorías que implican bastante actividad y energía y el menor número de comentarios congraciadores. Es congruente con tales pautas el que haya una clara tendencia en el bando perdedor a recibir más opiniones e información.

Como podía predecirse de la pauta antes comentada el bando ganador emitió una cantidad global de comentarios superior a la del bando perdedor.

También está de acuerdo con estos hallazgos un aspecto de los datos que consideramos especialmente revelador, pues señala que un criterio predictivo de influencia no es «hablar» sino un alegato activo en defensa de una posición establecida.

En el estudio de jurados anteriormente comentado, utilizamos un procedimiento experimental inspirado en Hoffmann y Maier (1964), denominado "valencia», mediante el cual codificamos cada comentario según fueran a favor de la acusación o de la defensa. Cuando reunimos los comentarios en pro de cada uno de los veredictos «inocente» y «culpable», respectivamente, sin tener en cuenta el emisor, hallamos que cuando la diferencia entre ambos montos era mayor de siete podían predecirse todos los veredictos de los treinta y siete grupos con excepción de uno. Las predicciones se efectuaban según el número de comentarios emitidos en pro de una determinada posición y no según el número de personas que hacían tales comentarios. Ello significa que la minoría precisa emitir doble cantidad de comentarios por persona para «seguir en la brecha». La defensa activa de su posición por parte de la minoría correlacionó con el éxito final de la posición minoritaria en aquellos casos en los que su número total de comentarios igualaba o excedía a los de la mayoría.

A partir de los resultados de investigación, que muestran la importancia de la firmeza de la posición y la argumentación activa en apoyo de las posturas propias, se plantea el problema de cómo lograr tal resistencia pasiva y persuasión activa. Muchos de los estudios de esta línea de investigación han utilizado cómplices del experimentador para evaluar los estilos conductuales y la importancia de la unanimidad. Nuestros estudios recientes confirman las conclusiones de aquéllos, aun en los casos en que las mayorías y minorías son bandos «naturales», es decir, todos son sujetos ingenuos que tienen puntos de vista diferentes.

La mayor parte de los bandos no suele actuar, sin embargo, en el vacío. Muchos tienen oportunidad de que sus miembros discutan entre ellos mismos y algunas observaciones recientes sobre el fenómeno de polarización sugieren una forma interesante de que uno u otro bando adquiera confianza y, además, según nuestras hipótesis, un mayor sentido de pertenencia al «grupo». Ello equivale a dar a cada bando de la situación experimental la oportunidad de discutir entre sí.

Uno de los fenómenos más interesantes y reproducibles que han estudiado los psicólogos sociales últimamente es el proceso de polarización. Mientras que, en un principio, la atención de los investigadores se centró en el «cambio arriesgado» y la investigación se dirigió a la comprensión de la aceptación de grandes riesgos, pronto se hizo evidente que una decisión arriesgada no era sino un ejemplo de un proceso más general, la polarización. Como sugiere una amplia y reciente revisión (Myers y Lamm, 1976), los hallazgos han sido bastante consistentes para cuestiones tan distintas como decisiones de un jurado, decisiones éticas, juicios, percepción interpersonal, negociaciones y decisión de riesgos. El fenómeno de polarización consiste en que «la respuesta post-grupo tenderá a ser más extrema en la misma dirección que la media de las respuestas previas a la situación de grupo" (Myers y Lamm, 1967, página 603). Una de las razones dadas para explicar tal fenómeno de polarización es que la discusión con otras personas que piensan del mismo modo aumenta la certidumbre; otra razón es que inicialmente 
los sujetos pueden atemperar sus puntos de vista esperando puntos de vista contrarios por parte de los demás (Walker y Main, 1973); además se aduce que «las influencias recíprocas de los miembros entre sí refuerzan y consolidan las orientaciones existentes» (Feldman $\mathrm{y}$ Newcomb, 1969, p. 223).

Debe subrayarse, sin embargo, que no todos los estudios realizados demostraron el fenómeno de polarización, algunos estudios no demostraron cambios significativos en la dirección predicha. Myers y Bach (1974), por ejemplo, hallaron que sujetos pacifistas y militaristas por separado devinieron más pacifistas tras una discusión con individuos que pensaban como ellos. Con todo, las evidencias son abrumadoramente acordes con la hipótesis de polarización incluso teniéndose en cuenta muchos tipos diferentes de toma de decisión.

Basándonos en este fenómeno, establecemos la hipótesis de que si una discusión previa por parte de un bando (sea de opinión mayoritaria o minoritaria) lleva a una polarización y un aumento de confianza en la opinión propia, tal discusión previa provocaría un mantenimiento de la posición propia y una mayor actividad en la defensa de dicha posición cuando ambos bandos se reúnen para tomar una decisión. Además, según nuestra hipótesis, tal discusión previa aumenta el sentido de pertenencia a un bando, un subgrupo. Como tales, los individuos pueden ser aún más renuentes a cambiar su opinión, puesto que ello puede percibirse como una deserción de un grupo o incluso, quizá, de una «causa». Aunque por diferentes razones, tales opiniones incrementarían la unanimidad, el mantenimiento de posiciones y a la larga, según nuestra hipótesis, la influencia.

$\mathrm{Si}$ el anterior proceso es resultado de la discusión previa, ello debería hacerse patente en la decisión definitiva a la que se llegue. Así, si se le da a la minoría la oportunidad de discutir previamente mientras que la mayoría no tiene esa oportunidad, la minoría debería resistirse a conformarse y a apoyar su postura con más seguridad. Así predeciríamos que la decisión resultante debería inclinarse en la dirección de la minoría. Del mismo modo, en el caso de que la mayoría haya discutido previamente y la minoría no, la decisión debería aproximarse a la mayoritaria. Si ambos o ninguno de los bandos tienen tal oportunidad, el veredicto (la decisión) debería estar a mitad de camino entre las decisiones previas de ambos bandos. Sin embargo, podemos predecir algunas diferencias entre la condición de «discusión previa en ambos bandos» $\mathrm{y}$ «sin discusión previa en ambos bandos».

Aunque ambas condiciones implican situaciones semejantes para los dos bandos, al menos en lo que concierne a la existencia o no de discusión previa, deberíamos esperar que en la condición de «no discusión previa» se llegue más fácilmente a una decisión que en la condición de «discusión en ambos bandos». Con la mayor implicación con el bando propio que crea la discusión previa puede llegarse a una situación de falta de voluntad para un compromiso o cambio de postura. Así, predecimos que cuando no se le da a ninguno de los bandos la oportunidad de una discusión previa, se tomará un mayor número de decisiones; en la condición de «discusión previa en ambos grupos» serían más las decisiones «pendientes». Sin embargo, no predecimos diferencias en el tipo de decisión tomada en ambas condiciones, en los casos en que se llegase a tomar una decisión.

El año pasado hemos realizado el estudio propuesto anteriormente. Si bien esperábamos reseñar en esta conferencia todos los resultados de este estudio, hemos concluido el registro de datos hace muy poco tiempo y en el momento de escribir estas líneas sólo disponemos de algunos análisis preliminares. Consideremos, en primer lugar, el procedimiento utilizado. El procedimiento supuso en el pretest que unos 1.200 estudiantes de la Universidad de California (Berkeley) evaluasen un conjunto de cuatro casos sobre daños a personas. En el caso que daba la división más clara en dos posibles veredictos, solicitábamos a las personas 
que obtasen entre un veredicto en favor de una indemnización de 100.000 dólares y otro en favor de una indemnización de 200.000 dólares para participar posteriormente en un experimento sobre toma de decisiones en jurados. De hecho, el mayor número de sujetos eligió 200.000 dólares como posición previa; la segunda opción, 100.000 dólares, fue adoptada por aproximadamente la mitad de los sujetos. Se distribuyó a los participantes de tal modo que en cada grupo cuatro personas apoyasen el veredicto de 200.000 dólares y dos el de 100.000 dólares. Así pudimos construir 40 grupos de seis personas cada uno, diez para cada una de las condiciones descritas anteriormente.

En la cita de cada grupo en una habitación común, los cuatro representantes de la posición mayoritaria eran conducidos a una habitación distinta y los de la posición minoritaria a otra. Ningún sujeto conocía las posiciones de los demás en este momento. Según la condición experimental se instruía a los bandos bien para que discutieran el caso durante quince minutos, examinando sus puntos de vista, o bien se les pidió simplemente que esperasen unos quince minutos mientras el experimento estuviera listo. A estos últimos se les pidió que no discutiesen ningún asunto entre sí. En una condición (sólo minoría) la minoría discutió previamente el caso y la mayoría sólo esperó; en la segunda condición experimental (sólo mayoría), la mayoría discutió el caso y la minoría esperó; en la tercera (ambos) los dos bandos discutieron el caso, y en la cuarta (ninguno) no se permitió discutir a nadie. Al final de este período de espera o discusión, los sujetos respondieron a un cuestionario muy breve dirigido fundamentalmente a averiguar su juicio personal y grado de seguridad.

A continuación se situó a todos los sujetos en una tercera habitación y se les pidió que deliberasen sobre el caso como si fuesen un jurado. Se les instruyó para que discutieran durante treinta y cinco minutos como máximo y que lle- garan a un veredicto unánime sobre la indemnización adecuada para el caso de daños y perjuicios personales planteado. Se grabó en video la discusión. Al llegar a un veredicto (o a los treinta y cinco minutos de discusión) se les pidió a los sujetos que respondieran a un cuestionario, más bien extenso, sobre su percepción del proceso e impresiones personales. Lo expuesto a continuación es sólo un análisis preliminar de los datos, pues éstos se codificaron sólo una semana antes de escribir esto.

Inicialmente, esperábamos que la «discusión previa» haría que la minoría adoptase la posición de 100.000 dólares o menos y la mayoría la de 200.000 dólares o más y que los bandos se sintiesen asegurados en su posición. Como señala la tabla 2, parece qué la discusión previa no hace que los bandos extremen sus juicios.

Todos los sujetos parecen haber incrementado la indemnización que consideraban adecuada en el pretest. Sin embargo, la discusión previa parece afectar al grado de confianza que cada bando tenía en su propia posición. Como muestra la tabla 3, el bando minoritario al que se permitió una discusión previa (en las condiciones experimentales «sólo minoría» y «ambas») parece estar más seguro de sí mismo que la minoría a la que no se le permitió la discusión («sólo mayoría» y «ninguno»). Del mismo modo, el bando mayoritario al que se le permitió la discusión («sólo mayoría» y «ambos») parece más seguro que la mayoría a la que no se le permitió la discusión («sólo minoría» y «ninguno»).

Con respecto al veredicto final, nuestra hipótesis de que las condiciones experimentales «ambos»y «ninguno» diferían en lo que respecta al número de decisiones logradas parece confirmarse. Como se observa en la tabla 4 , la condición experimental «ambos» implica el mayor número de jurados «pendientes de veredicto». Le sigue muy de cerca la condición experimental «sólo minoría». Por otra parte, se tomó el mayor número de decisiones cuando la mayoria 
había discutido previamente, pero la minoría no. Como se apreciará en la tabla 3, la mayoría más segura de sí misma y la minoría más insegura aparecen en esta última condición; quizá ello sea la razón por lo que los veredictos se emitieron, habitualmente, en la línea mayoritaria.

TABLA 2

Posición privada tras la discusión previa

\begin{tabular}{lccccc}
\hline & $\begin{array}{c}\text { Sólo } \\
\text { minoría }\end{array}$ & $\begin{array}{c}\text { Sólo } \\
\text { mayoría }\end{array}$ & Ambos & Ninguno & $\overline{\mathrm{X}}$ \\
\hline Sujetos minoría & 114.150 & 115.000 & 116.800 & 118.400 & 116.088 \\
Sujetos mayoría & 213.475 & 224.250 & 216.775 & 184.650 & 212.288 \\
\hline
\end{tabular}

TABLA 3

Confianza (a) en la posición privada tras la discusión previa

\begin{tabular}{lccccc}
\hline & $\begin{array}{c}\text { Sólo } \\
\text { minoría }\end{array}$ & $\begin{array}{c}\text { Sólo } \\
\text { mayoría }\end{array}$ & Ambos & Ninguno & $\overline{\mathrm{X}}$ \\
\hline Minoría & 2,2 & 3,3 & 2,2 & 2,7 & 2,6 \\
Mayoría & 1,8 & 1,2 & 1,7 & 2,3 & 1,8 \\
\hline
\end{tabular}

(a) La confianza se califica en una escala de siete puntos, siendo uno «muy seguro» y 7 «muy inseguro».

TABLA 4

\begin{tabular}{lcccc}
\hline & $\begin{array}{c}\text { Sólo } \\
\text { minoría }\end{array}$ & $\begin{array}{c}\text { Sólo } \\
\text { mayoría }\end{array}$ & Ambos & Ninguno \\
\hline Veredicto & 4 & 8 & 3 & 6 \\
$\begin{array}{l}\text { Pendiente } \\
\begin{array}{l}\text { Indemnización en los grupos que se } \\
\text { pusieron de acuerdo }\end{array}\end{array}$ & 6 & 2 & 7 & 4. \\
\hline
\end{tabular}

El monto de la indemnización acordada por los grupos que lograron el consenso es menos concluyente. Como puede observarse en la tabla 4, la indemnización no parece diferir demasiado según las oportunidades de discutir. Sería, sin embargo, prematuro especular sobre estos hallazgos, puesto que se basan en grupos muy específicos y aguardan un análisis más detallado de los datos del cuestionario, así como la codificación de las intetacciones entre los miembros de la mayoría y minoría.

En este estudio buscamos un alto grado de regularidad en los procesos de influencia y la hipótesis debe explorarse en base a variables dependientes establecidas previamente. Supusimos una polarización de la opinión en términos del monto de indemnización considerado adecuado según las oportunidades de discusión. Los datos indican, sin embargo, un 
incremento en «el juicio de indemnización» tanto para los sujetos mayoritarios como para los minoritarios. Ello puede deberse a los valores sociales relacionados con las compensaciones para víctimas de daños y las consideraciones éticas que se hayan tenido en cuenta durante la discusión. También predijimos que un aumento de seguridad respecto a la posición defendida dependería de las oportunidades de discusión. Esto parece haberse confirmado con nuestros datos. Los bandos a los que se les permite una discusión previa parecen estar más seguros de sus juicios.

La clave argumental radica, sin embar. go, en que tal aumento de seguridad debe manifestarse en unos estilos conductuales que crean las percepciones de consistencia y seguridad. También debería observarse en la resistencia a las tácticas persuasivas del otro bando. Tales aspectos se comprobarán por medio de un análisis de las pautas de interacción registradas en video. Los estilos interactivos facilitarán la comprensión de las causas de que se llegue o no a una decisión y la dirección de la decisión cuando haya acuerdo.

Aunque nuestras hipótesis sean relativamente sencillas, es obvio para los que trabajan sobre la interacción entre mayorías y minotías naturales que los procesos son muy complejos. Aun cuando sean correctas nuestras premisas sobre la importancia de la resistencia y la argumentación activa, y aun cuando sean correctas nuestras premisas sobre la eficacia de la discusión previa para la manipulación de la seguridad, puede darse el caso de que una mayor seguridad en los propios juicios no siempre se manifieste en estilos conductuales adecuados. Nuestro reto más importante es la exploración de este proceso.

Como punto de partida creo que los principios sobre la estabilidad son un modo apropiado de considerar las pautas de influencia entre mayorías y minorías. Sin embargo, al plantearlo así nos circunscribimos a las similitudes entre los procesos de conformidad e influencia minoritaria, compromiso y polarización. Con todo, el tamaño del bando, o mejor dicho, la noción de pertenecer a una mayoría o a una minoría tiene, como hemos señalado, consecuencias. Nuestros datos nos recuerdan que los procesos son distintos para ambos bandos y que el proceso de toma de decisión es, una vez más, más complejo que nuestras teorías.

\section{Referencias bibliográficas}

Ascr, S. E.: Social Psychology. Englewood Cliffs, N. J.: Prentice-Hall, 1952.

Asch, S. E.: Opinions and social pressure. Scientific American, 1955, 193, 31-35.

Asch, S. E.: Studies of independence and conformity: A minority of one against a unanimous majority. Psychological Monographs, 1956, 7 (9, Whole No. 416).

Deutsch, M., y Gerard, H. B.: A study of normative and informational social influences upon individual judgement. Journal of Abnormal and Social Psychology, 1955, 51, 629-636.

Feldman, R. A., y Newcomb, T.: The impact of college on students. San Francisco: JosseyBass, 1969.

GoldBerg, S. C.: Three situational determinants of conformity to social norms. Journal of Abnormal and Social Psychology, 1954, 49, 325-329.

Hoffman, R., y Naier, Norman, R. F.: Valence in the adoption of solutions by problemsolving groups: Concept, method and results. Journal of Abnormal and Social Psychology, $1964,69,264-271$.

Kalven, Harry JR., y Zeisel, Hans: The American Jury. Boston: Little, Brown \& Co., 1966.

Moscovici, S.; Lage, E., y Naffrechoux, M.: Influence on a consistent minority on the responses of a majority in a color perception task. Sociometry, 1969, 32, 365-380.

Myers, D. G., y Lamm, H.: The group polarization phenomenon. Psychological Bulletin, 1976, $83,602-627$.

Nemeth, C.: A comparison between conformity and minority influence. Paper presented to International Congress on Psychology, París, France, July 1976. 
NemETH, C.: Interactions between jurors as a function of majority $\mathbf{v}$. unanimity decision rules. Journal of Applied Social Psychology, 1977, 7, 38-56.

NemEth, C.: The role of an active minority in inter-group conflict. En W. G. Austin and S. Worchel (Eds.). The Psychology of Intergroup Relations. Belmong, Cal.: Brooks-Cole Publishing Co., 1978, en prensa.

Nemeth, C., y WACHTLER, J.: Creating the perceptions of consistency and confidence: A necessary condition for minority influence. Sociometry, 1974, 37, 529-540.

Nemeth, C., y WaChTLeR, J.: Creative problem solving as a function of majority vs minority influence. Submitted to Journal of Personality and Social Psychology, 1981.

Nemeth, C.; Swedlund, M., y KanKi, B.: Patterning of the minority's responses and their influence on the majority. European Journal of Social Psychology, 1974, 4, 53-64.

Nemeth, C.; Endicot', J., y Wachtler, S.: From the '50s to the '70s: Women in jury deliberations. Sociometry, 1976, 39, 293-304.

Rosenberg, L. A.: Group size, prior experience and conformity. Journal of Abnormal and Social Psychology, 1961, 63, 436-437.

SCHACTER, S.: Deviation, rejection and communication. Journal of Abnormal and Social Psychology, 1951, 46, 190-207.

Walker, T. G., y MaIN, E. C.: Choice-shifts in political decision making: Federal judges and civil liberties cases. Journal of Applied Social Psychology, 1973, 2, 39-48. 